\title{
Is the Hubble Constant the same everywhere in the Universe?
}

\author{
Jaroslav Hynecek ${ }^{1}$ \\ ${ }^{1}$ Isetex, Inc., 905 Pampa Drive, Allen, TX 75013, USA \\ Correspondence: Jaroslav Hynecek, Isetex, Inc., 905 Pampa Drive, Allen, TX 75013, USA. E-mail: \\ jhynecek@netscape.net
}

Received: April 16, 2015 Accepted: April 27, 2015 Online Published: April 28, 2015

doi:10.5539/apr.v7n3p60

URL: http://dx.doi.org/10.5539/apr.v7n3p60

\begin{abstract}
This paper describes the derivation of formula for the galaxies' recession velocities in the finite size model of the Universe. From this formula it is then derived the Hubble constant dependency on the distance from the Universe's center, which is relatively near the Milky Way galaxy. It is shown that for this model of the Universe the Hubble constant for very distant objects is significantly reduced and, therefore, shouldn't be measured and evaluated using the standard methods without corrections such as, for example, in gravitational lensing when such large distances and consequently the large redshifts are involved. The Hubble constant in this Universe is also independent of time.
\end{abstract}

Keywords: galaxy redshift, finite size model of the Universe, Big Bang theory, gravitational redshift, Doppler redshift, Hubble constant, deformable dark matter, cosmological gravitational potential, gamma ray bursts, CMBR temperature

\section{Introduction}

There are many papers published in the literature on the Hubble constant and its measurements. A comprehensive reviews can, for example, be found in (Lauer et al., 1997) or in a more recent publication by (Freedman et al., 2000). This large amount of work, however, produced the values of Hubble constant with only $10 \%$ accuracy. Another problem is that all this effort is based on the models of the Universe that are derived from the Big Bang (BB) assumption. This leads to a certain bias in the evaluation of data, which may lead to erroneous conclusions. For example: it is always assumed that the Hubble constant is a true constant and that it has not changed in time since the beginning of the Universe and therefore it is the same everywhere in the Universe. This may not be true and would obviously lead to problems when the Hubble constant is evaluated for large distances as for example in (Lauer et al., 1997). The current paper addresses these points and derives the Hubble constant dependency on the distance in a model of the Universe that is not based on the BB assumption.

In the previous publication Hynecek (2012a) has introduced a new model of the Universe where it is assumed that the Universe is finite in size and filled with a repulsive and deformable Dark (transparent) Matter (DM). The DM is repulsive to visible matter but attractive to itself. In this model the galaxies are treated only as small test bodies floating from the bulk of the Universe to the edge where they explode and generate the well-known immense Gamma Ray Bursts (GRB) detected here on Earth. The GRBs that are reflected back to the bulk of the Universe then contribute to the generation of the new matter. This model is in line with the theory proposed by Hoyle, Burbidge \& Narlikar (2000) of a steady state Universe with a constant matter creation. The new matter then condenses to stars and eventually to new galaxies endlessly repeating the cycle of creation and destruction. The galaxies' explosions residue at the edge of the Universe generates also the Cosmic Microwave Background Radiation (CMBR) with its temperature of $2.72548{ }^{\circ} \mathrm{K}$. The repulsive DM density is very small but its gravitational effects dominate the visible matter gravitation at large distances. The gravitational field of galaxies is thus compensated and screened by the DM after a certain distance. The galaxies thus for the most part move in this Universe independently of each other.

The one of the significant contributions of this model to the theory of the Universe, in comparison to other models of the Universe in particular the BB model, is in the derivation of the relation between the Hubble constant and the CMBR temperature (Hynecek, 2013). These two parameters are typically considered independent of each other. In this model they have been found dependent and one can be derived from the other. This is only possible in a finite and thermodynamically enclosed model as this one is and not in the open models such as the BB. 
The details of the model mathematical background and its excellent agreement with the various observations have been published earlier by Hynecek (2012a, 2014). The important equations needed for the derivation of the Hubble constant dependency on the distance are presented in the next section.

\section{The Hubble Constant Dependency on the Distance from the Center of the Universe}

In a previous publication Hynecek (2015) has presented the formula for the recession velocity of galaxies as follows:

$$
\frac{d r}{d t}=c e^{2 \varphi_{v}} \sqrt{1-e^{2 \varphi_{v}}}
$$

where the gravitational potential for the visible matter normalized to $c^{2}$ is calculated using the standard Newton-like relation:

$$
\varphi_{v}(r)=-\frac{4 \pi \kappa}{c^{2} \rho(r)} \int_{0}^{\rho(r)} m(\rho) \rho(r)^{2} d \rho
$$

In this formula $\kappa$ is the gravitational constant, $c$ the speed of light, and the physical radius $\rho(r)$ has been substituted for the natural radius $r$ due to the natural radius being distorted by the DM gravity.

Once the gravitational potential for the visible matter is found the Hubble constant dependence on the distance can easily be evaluated following the standard definition:

$$
H_{u}(r)=\frac{d \ln (r)}{d t}=\frac{c}{r} e^{2 \varphi_{v}} \sqrt{1-e^{2 \varphi_{v}}}
$$

For the reader's convenience a shortened version of derivations presented in the previously published paper (Hynecek, 2015) is given in the Appendix. The previous paper has also presented the formula for the redshift $Z$ dependence on the cosmological gravitational potential for the visible matter as follows:

$$
Z(\rho)=\frac{\sqrt{1-v_{r}^{2} / c_{r}^{2}}}{1-v_{r} / c_{r}}-1=\frac{\sqrt{g_{t t}}}{1-\sqrt{1-g_{t t}}}-1=\frac{\exp \left(\varphi_{v}\right)}{1-\sqrt{1-\exp \left(2 \varphi_{v}\right)}}-1
$$

For a more convenient evaluation, once the $Z$ dependence on the radial distance $r$ is known, it is helpful to express the galaxies' recession velocity and the speed of light as functions of the redshift $Z$, because this parameter is actually what is measured by astronomers. For this purpose it is useful to derive first the cosmological gravitation potential for the visible matter as a function of the redshift $Z$ using Equation 4. The result is as follows:

$$
\exp \left(\varphi_{v}\right)=\frac{2(Z+1)}{(Z+1)^{2}+1}
$$

From this result the formulas for the radial speed of light $c_{r}$ and the galaxies' recession velocity $v_{r}$, as observed from Earth, can be expressed as follows:

$$
\begin{gathered}
c_{r}(Z)=c g_{t t}=c\left(\frac{2(Z+1)}{(Z+1)^{2}+1}\right)^{2} \\
v_{r}(Z)=c_{r}(z) \frac{(Z+1)^{2}-1}{(Z+1)^{2}+1}
\end{gathered}
$$

These dependencies are plotted in graphs in Figure 1 as functions of the natural radial distance $r$.

For more clarity these two parameters are plotted again as functions of the redshift $Z$ in Figure 2. This plot is expanding the region of distances that are greater than one billion light years. It is interesting to observe from these graphs that the galaxies' recession velocity is approaching the speed of light as they approach the border region of the Universe. The galaxies' recession velocity reaches approximately $98.5 \%$ of the speed of light in that region.

Once the galaxies' recession velocity dependence on the cosmological gravitational potential is known it is simple to use the formula in Equation 3 and plot the Hubble constant dependency on the natural radial distance from the center of the Universe. This is shown in Figure 3 together with the redshift $Z$ dependency on the same distance. The graphs in Figures 1 and 3 should terminate at the maximum universe radius: $r_{m x}=22.11 \cdot 10^{9} \mathrm{Ly}$, because there is no spacetime past that distance. The graphs continuation is only an artifact of calculations. The coordinates that extend past the maximum radius actually do not describe any physical entity. 
Finally, for additional clarity, the Hubble constant dependency on the redshift $Z$ is shown in a graph in Figure 4 . From this result it is clear that the Hubble constant remains the same up to approximately $Z=0.1$, but for the redshifts larger than $Z>0.1$ the measurements or observations require significant corrections due to the curvature of the Universe. The curvature starts to have a significant effect after the distance of one billion light years.

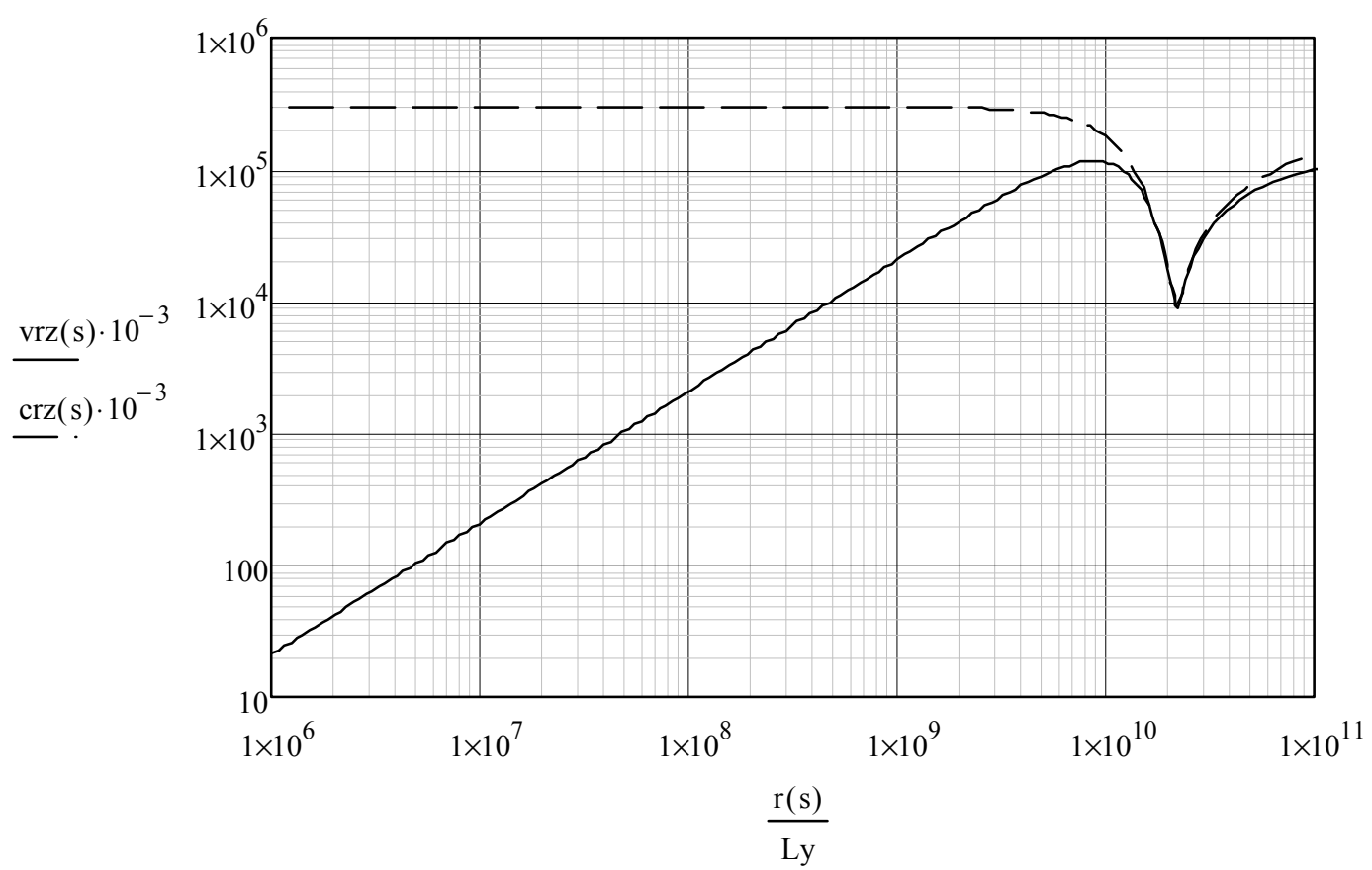

Figure 1. The graphs of the light speed (dashed trace) and the galaxies' recession velocity as functions of distance from the Universe's center measured in light years. There is no space-time beyond the maximum Universe radius

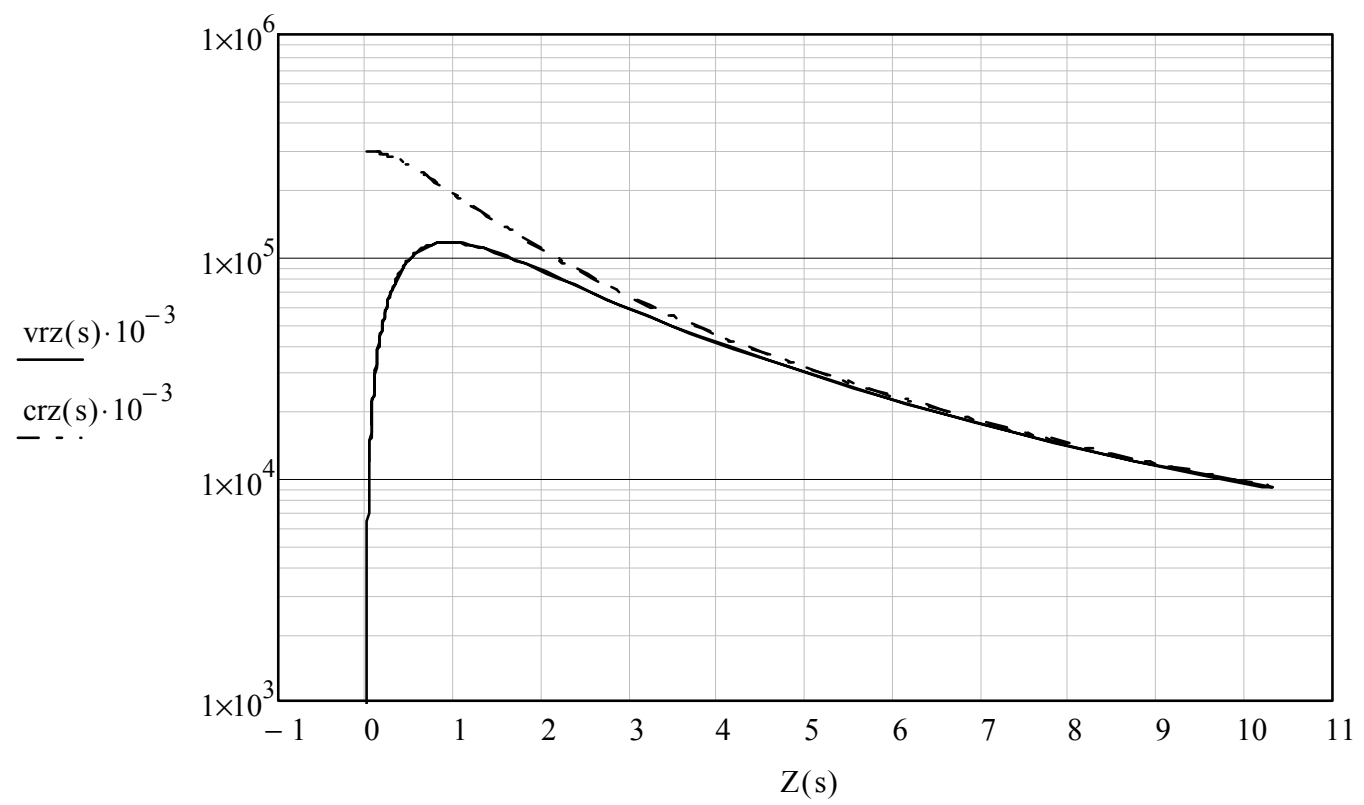

Figure 2. The graphs of the light speed (dot dashed trace) and the galaxies' recession velocity plotted as functions of the redshift $Z$. There is no space-time beyond the maximum Universe's radius at $Z=10.35$. For a better clarity the $Z$ axis has been started from -1 instead of from zero 


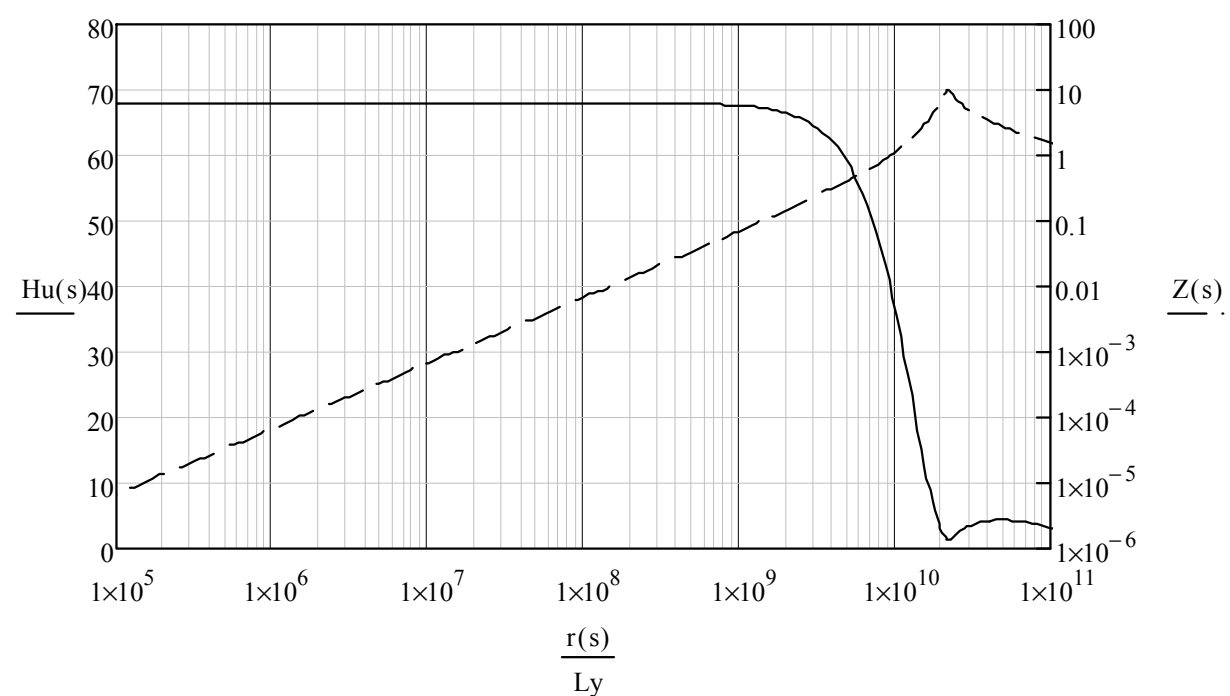

Figure 3. The graphs of the Hubble constant dependency and the redshift $Z$ dependency (dashed trace) on the natural radial distance $r$ from the center of the Universe measured in light years

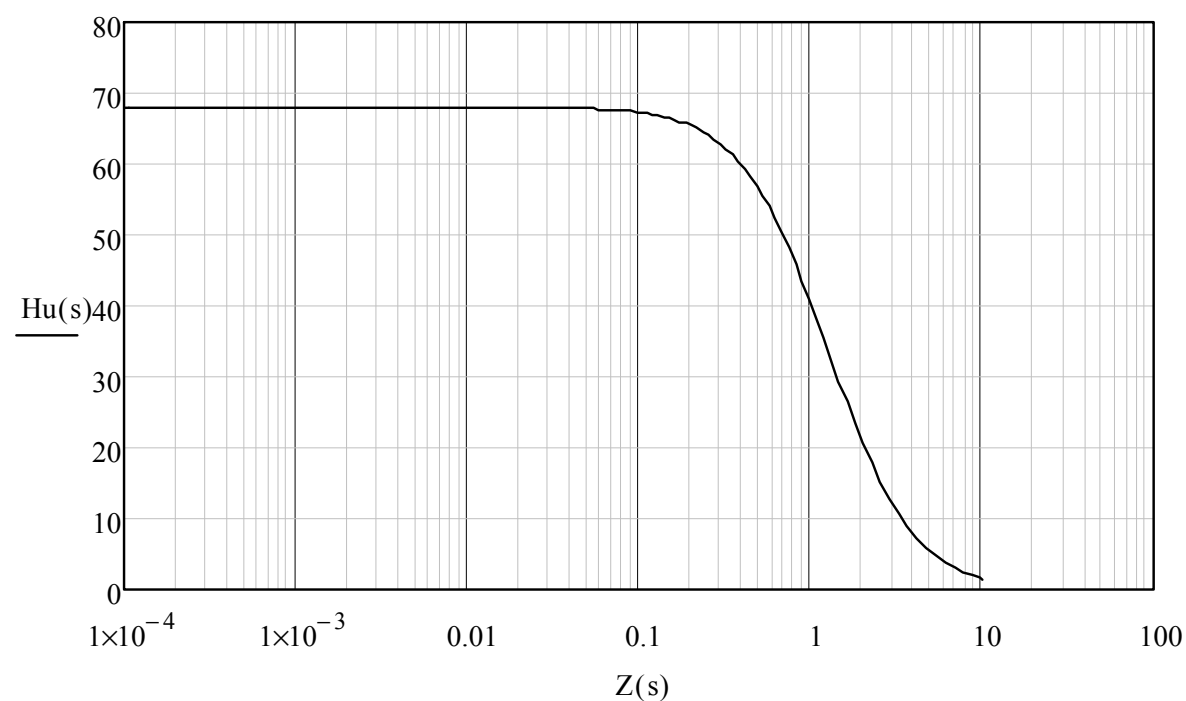

Figure 4. The graph of the Hubble constant dependency on the redshift $Z$

The Hubble constant used in all these calculations was: $H_{0}=68.0 \mathrm{~km} / \mathrm{secMpc}$. This value is in an excellent agreement with the value derived by Hynecek (2012a, 2013) from the very precisely measured CMBR temperature: $T_{0}=2.72548 \pm 0.00057^{\circ} \mathrm{K}$ (Fixsen, 2009). The formula derived in the previous publication (Hynecek, 2013) is relating these two parameters as follows:

$$
H_{0}=\frac{8}{3} \sqrt{\frac{\pi \kappa}{c^{5} h^{3}}}\left(\frac{k_{B} T_{0}}{\exp \left(2 \varphi_{v}\right)}\right)^{2}=67.920 \mathrm{~km} / \mathrm{sec} M p c
$$

where $k_{B}$ is the Boltzmann constant, $h$ is the Planck constant, and the visible matter cosmological gravitational potential: $\varphi_{v}=-1.7436$ is found at the Universe's edge using Equation 2 and Equation a13 shown in Appendix. This formula provides a much more accurate value for the Hubble constant than the one found in the recent literature (Planck Mission, 2015) and can be used, as one among the several others (Hynecek, 2012a), as the direct evidence in support of the finite size Universe theory.

\section{Other Definitions of Hubble Constant}

The derivations of Hubble constant dependency on the distance in previous section of the paper were all based on the one particular definition given in Equation 3. Other definitions are certainly possible leading to the same value 
of $H_{0}$ at the center of the Universe, near Earth. For example: the recession velocity could be normalized by the radial speed of light and by the ratio of physical radius to natural radius, since the radial speed of light and the natural radius are both reduced at large distances. This leads to the following formula for the Hubble constant $H_{\mathrm{n}}(Z)$ that will be called the normalized version:

$$
H_{n}(Z)=\frac{c \rho}{c_{r} r} \frac{d \ln (r)}{d t}=\frac{c \rho(r)}{r^{2}} \sqrt{1-\exp \left(2 \varphi_{v}\right)}
$$

Another version of the Hubble constant formula definition could, for example, use the luminance distance defined as: $d_{l u}=\rho(r)(Z+1)$ leading to the result:

$$
H_{l u}(Z)=\frac{d \ln (\rho(Z+1))}{d t}=c \exp \left(\varphi_{v}\right)\left(\frac{\sqrt{1-\exp \left(2 \varphi_{v}\right)}}{\rho(r)}-\frac{d \varphi_{v}}{d \rho(r)}\right)
$$

The comparison between the standard Hubble constant dependency on the redshift $Z$, the normalized version definition dependency, and the luminance distance version definition dependency are shown in Figure 5.

As can be seen from the graphs the normalized Hubble constant provides results that say the same as at the center of the Universe to larger redshifts. For the luminance distance version the values at larger distances grow higher sooner compared to the values at the Universe's center. This could explain the larger than 80 values of the Hubble constant found for the far field (Lauer et al., 1997) and could lead to a wrong conclusion that the Universe's expansion is accelerating. It is also possible to devise other definitions with correction factors that result in different values of $H(Z)$ at large distances. It is therefore necessary to always extrapolate the measurements and observations to the center of the Universe to obtain the correct values for the Hubble constant without large errors.

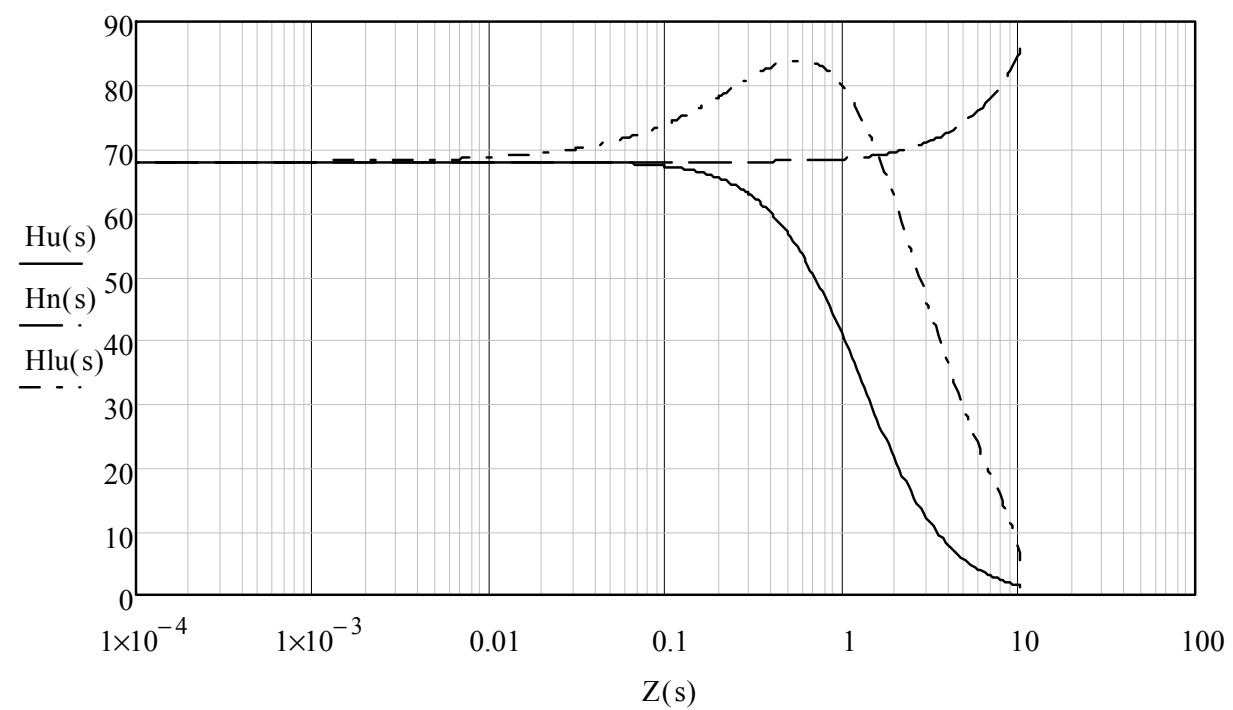

Figure 5. The graphs are comparing the standard definition of Hubble constant dependency on the redshift $Z$, the normalized definition of Hubble constant dependency (dashed trace), and the luminance distance definition of Hubble constant dependency (dot dashed trace) all on the same redshift $Z$. The maximum redshift is: $Z_{\mathrm{mx}}=10.35$

\section{Conclusions}

The article described detail calculations of Hubble constant dependency on the natural radial distance from the center of the Universe. It was found that the value of the constant evaluated in the vicinity of Earth for the nearby objects is significantly reduced when the observed objects have redshifts larger than $Z>0.1$. This is important when the Hubble constant is evaluated for the far fields, for example, when using the gravitational lensing. For such large distances it is necessary to include the curvature of the Universe into considerations. Possible corrections to the Hubble constant definition were explained in section $\mathbf{3}$ and the results compared to the standard definition values. The normalized Hubble constant definition provides values that stay constant to larger redshifts.

The model of the Universe, which was used for the calculation of Hubble constant dependency on the distance, is finite in size, it is static, and only the galaxies move in it from the bulk to the border regions where they explode and generate the well-known GRBs. As a consequence of the static model of the Universe the Hubble constant remains unchanged with the elapsed time of observations. This is not the case for the BB model as discussed for, 
example, by Xu (2009). The evidence in support of the veracity of static and finite size model of the Universe is in the derivation of Hubble constant from the CMBR temperature. The previously derived and published formula for the Hubble constant yields an excellent agreement with observations.

\section{Final Comments}

The author of this paper believes that the static and spatially finite model of the Universe is superior to the BB model, which was constructed by a simplistic extrapolation of observations of receding galaxies. The static and spatially finite model has fewer problems of satisfying the basic and well established laws of physics, such as the conservation of mass and energy. Obviously, the model has no problems with the initial singularity of the BB model where the laws of physics are failing and must be unnaturally suspended. The static and spatially finite model of the Universe avoids the peculiarity of creating something from nothing and in addition is keeping the fundamental constants of physics independent of time. Finally, it is also obvious that the Universe that is filled with the DM of however small density, which is supporting the propagation of light as transversal waves and the gravity as longitudinal waves, must necessarily be finite in size. The infinite size of such Universe would produce an infinite DM pressure and consequently the infinite speed of light following the formula: $c=\sqrt{P / m}$. This would contradict experimental facts and lead to many other well-known consequences, for example, in Maxwell equations where it holds that: $\varepsilon_{0} \mu_{0}=1 / c^{2}$. The Universe without a Big Bang was recently proposed by Ali and Das (2014).

\section{References}

Ali, A. F., \& Das, S. (2015). Cosmology from quantum potential. Physics letters B, 741, 276-279. http://dx.doi.org/10.1016/j.physletb.2014.12.057

Fixsen, D. J. (2009). The Temperature of the Cosmic Microwave Background. The Astrophysical Journal, 707(2), 916-920. http://dx.doi.org/10.1088/0004-637X/707/2/916

Freedman, W. L., Madore, B. F., Gibson, B. K., Ferrarese, L., Kelson, D. D., Sakai, S., ... \& Stetson, P. B. (2001). Final results from the Hubble Space Telescope key project to measure the Hubble constant. The Astrophysical Journal, 553(1), 47. http://dx.doi.org/10.1086/320638

Hoyle, F. G., Burbidge, F. G., \& Narlikar, J. V. (2000). A Different Approach to Cosmology. (Cambridge, U.K.: Cambridge University Press)

Hynecek, J. (2011). Geometry based critique of general relativity theory, Physics Essays, 24(2), 182. Retrieved from http://www.highbeam.com/doc/1G1-258052469.html

Hynecek, J. (2012a). Repulsive dark matter model of the universe. Physics Essays, 25(4), 561. http://dx.doi.org/10.4006/0836-1398-25.4.561_

Hynecek, J. (2012b). The Theory of Static Gravitational Field, Applied Physics Research, 4(4), 44-57. http://dx.doi.org/10.5539/apr.v4n4p44

Hynecek, J. (2013). The Repulsive Dark Matter Model of the Universe Relates the Hubble Constant to the Temperature of the CMBR. Applied Physics Research, 5(2), 76-83. http://dx.doi.org/10.5539/apr.v5n2p7

Hynecek, J. (2014). An Interesting Journey of Discovery of Many Errors in Einstein's General Relativity Theory. Retrieved from http://vixra.org/abs/1407.0136

Hynecek, J. (2015). On the Detection Possibility of Extragalactic Objects' Redshift Change. Applied Physics Research, 7(2), 58-65. http://dx.doi.org/10.5539/apr.v7n2p58

Lauer, T. R., Tonry, J. L., Postman, M., Ajhar, E. A., \& Holtzman, J. A. (1998). The Far-Field Hubble Constant. The Astrophysical Journal, 499(2), 577. http://dx.doi.org/10.1086/305671

Planck Mission. (2015). In Wikipedia, the free encyclopedia. Retrieved from http://en.wikipedia.org/wiki/ Planck_(spacecraft)\#2013_data_release

Xu, F. (2009). Apparent Time-Dependence of the Hubble Constant Deduced from the Observed Hubble Velocity-Distance Equation. Retrieved from http://vixra.org/pdf/0912.0016v1.pdf

Zel'dovich, Ya. B., and Novikov, I. D. (2011). Stars and Relativity. NY: Dover Publications, Inc.

\section{Appendix: mathematical background}

Since the long range gravitational effects of the visible mater and all of the radiation can be neglected, the space-time metric can be considered static, spherically symmetric, and described by the following differential metric line element (Hynecek, 2011, 2012b): 


$$
d s^{2}=g_{t t}(c d t)^{2}-g_{r r} d r^{2}-\rho^{2} g_{t t} d \Omega^{2}
$$

where: $d \Omega^{2}=d \vartheta^{2}+\sin ^{2} \vartheta d \varphi^{2}, g_{t t}=\exp \left(2 \varphi_{v}\right), g_{t t} g_{r r}=1$, and $c$ is the local intergalactic speed of light. The cosmological Newton-like gravitational potential for the visible matter $\varphi_{v}$ normalized to $c^{2}$ is calculated using the well-known equation:

$$
\varphi_{v}(r)=-\frac{4 \pi \kappa}{c^{2} \rho(r)} \int_{0}^{\rho(r)} m(\rho) \rho(r)^{2} d \rho
$$

where $\kappa$ is the Newton gravitational constant. Due to the deformation of the observed natural radius $r$ by the DM gravity, the physical radius $\rho(r)$ must be used in the formula instead of $r$ and this parameter is found from the differential equation that follows from the metric:

$$
d \rho=\sqrt{g_{r r}} d r=e^{-\varphi_{v}} d r
$$

Since any particular galaxy now represents only a small test body in this Universe, the well-known and many times verified Lagrange formalism can be used to describe the motion of such galaxies. The Lagrangian is therefore as follows:

$$
L=e^{2 \varphi_{v}}\left(\frac{c d t}{d \tau}\right)^{2}-e^{-2 \varphi_{v}}\left(\frac{d r}{d \tau}\right)^{2}-\rho^{2} e^{2 \varphi_{v}}\left(\frac{d \Omega}{d \tau}\right)^{2}
$$

For a purely radial motion the Lagrangian can be simplified and the first integrals of the corresponding Euler-Lagrange equations easily found using the initial condition at the origin where the recession velocity is zero and where: $d \tau=d t$. The results are:

$$
\begin{gathered}
\frac{d t}{d \tau}=e^{-2 \varphi_{v}} \\
\left(\frac{d r}{d \tau}\right)^{2}=c^{2}-c^{2} e^{2 \varphi_{v}}
\end{gathered}
$$

Eliminating the non-observable $d \tau$ from these equations then leads to the formula for the recession velocity:

$$
\frac{d r}{d t}=c e^{2 \varphi_{v}} \sqrt{1-e^{2 \varphi_{v}}}
$$

For the relatively near objects, where the cosmological gravitational potential for the visible matter $\varphi_{v}$ is still small, it holds that: $\rho(r) \sim r$, and $m(\rho)=m_{0}$. This simplifies Equations a2 and a7 leading to the following formula:

$$
\frac{d r}{d t} \cong c \sqrt{-2 \varphi_{v}}=\sqrt{\frac{8}{3} \pi \kappa m_{0}} r=H_{0} r
$$

From this result it is then clear that the recession velocity is linearly proportional to the natural coordinate distance $r$ of such nearby objects from the origin and that the Hubble constant $H_{0}$ is related to the DM density $m_{0}$ at the origin according to the following Equation:

$$
H_{0}=\sqrt{\frac{8}{3} \pi \kappa m_{0}}
$$

The recession velocity and the Hubble constant are referenced to the DM coordinate system, so the value of the Hubble constant should be corrected and referenced to the Earth's centered coordinate system from where it is actually measured. However, the correction is very small and it will be neglected. Earth and its Milky Way galaxy are located relatively near the center of the Universe in comparison to its immense size.

It is therefore clear that the Hubble constant $H_{0}$ should be measured only for the nearby objects and not assumed that it is the same everywhere in the Universe. The dependency on the radial distance can easily be derived from Equation a7 using the Hubble constant defining formula shown below. Other definitions are discussed in section 3.

$$
H_{u}(r)=\frac{d \ln (r)}{d t}=\frac{c}{r} e^{2 \varphi_{v}} \sqrt{1-e^{2 \varphi_{v}}}
$$

In order to proceed further in the derivations it is necessary to find the cosmological gravitation potential for the visible matter and therefore the relation for the DM density $m(\rho)$ as a function of the physical radius. This is 
obtained by adapting the well-known approach described, for example, by Zel'dovich and Novikov (2011) where the DM pressure gradient is expressed as a function of the physical radial distance:

$$
\frac{d P}{d \rho}=-\frac{4 \pi \kappa m(\rho)}{\rho^{2}} \int_{0}^{\rho} m(\rho) \rho^{2} d \rho
$$

After substituting for the DM pressure the relation: $P=m c^{2}$, which is equivalent to: $c=\sqrt{P / m}$, and defining the normalized mass density: $m_{n}(\rho)=m(\rho) / m_{0}$, Equation 11 can be rearranged with the help of Green's function as:

$$
m_{n}(\rho)=\exp \left(-A_{0} \int_{0}^{\rho} m_{n}(\xi)\left(\xi-\xi^{2} / \rho\right) d \xi\right)
$$

where $A_{0}$ is a constant equal to: $A_{0}=4 \pi \kappa m_{0} / c^{2}$. There is no known analytic closed form solution for this equation, so it is necessary to use the numerical iterative approach or find an approximating function. The approximating function approach was selected for the next steps to avoid very long computing times during iterations. The selected function, however, underestimates the true value of the DM mass density at large $\rho$, but the error has only a small overall effect. The approximating function is as follows:

$$
m_{a}(\rho)=\exp \left(-\frac{\rho^{2}}{\rho_{h}^{2}}+\frac{3}{10} \frac{\rho^{4}}{\rho_{h}^{4}}-\frac{4}{35} \frac{\rho^{6}}{\rho_{h}^{6}}+\frac{61}{1260} \frac{\rho^{8}}{\rho_{h}^{8}}-\frac{4507}{231000} \frac{\rho^{10}}{\rho_{h}^{10}}+\ldots\right)
$$

The introduced parameter defined as: $\rho_{h}=2 c / H_{0}$, is called the Hubble distance or the Hubble physical radius.

Another advantage of using the approximating function is that the DM concentration tail extending past the maximum radial distance can be easily cut off by suitably truncating the power series expansion in the exponent. This feature is advantageous if it is considered that the visible matter debris from explosions of galaxies are accumulating at the edge of the universe and are forming a loosely bound shell there.

Once the mass density function is known it is easy to find the normalized gravitational potential for the visible matter using the formula in Equation 2, and for the dark matter using the Green's function formula derived also from the Gauss law as follows:

$$
\varphi_{d}(\rho)=A_{0} \int_{0}^{\rho} \frac{d \xi}{\xi^{2}} \int_{0}^{\xi} m_{a}(\zeta) \zeta^{2} d \zeta-3.303=A_{0} \int_{0}^{\rho} m_{a}(\xi)\left(\xi-\xi^{2} / \rho\right) d \xi-3.303
$$

Both potentials are plotted in the graphs shown in Figure a1.

In the next step of the model description it is necessary to find the formula for the $Z$ shift, since this is the parameter that is directly measured by astronomers. The $Z$ shift typically consists of the three components: the star gravity induced redshift, the cosmological potential induced redshift, and the Doppler redshift resulting from the recession velocity. The star gravity intrinsic redshift does not have to be considered, since after the star or the galaxy explosion have occurred, when the Supernova explosions or the GRB data are compiled, most of the principal source of the gravitational field has been converted to radiation and radiated away and only the remnants or the afterglow produce the light that is observed. The cosmological potential induced redshift does not have to be considered either, since in this model the galaxies are in a radial free fall and this compensates for the shift.

The only remaining redshift component is thus the Doppler redshift resulting from the radial recession velocity $v_{r}$. The Doppler redshift observed on Earth, therefore, is:

$$
Z(\rho)=\frac{\sqrt{1-v_{r}^{2} / c_{r}^{2}}}{1-v_{r} / c_{r}}-1=\frac{\sqrt{g_{t t}}}{1-\sqrt{1-g_{t t}}}-1=\frac{\exp \left(\varphi_{v}\right)}{1-\sqrt{1-\exp \left(2 \varphi_{v}\right)}}-1
$$

where $c_{r}$ indicates the light speed at the galaxy location in reference to Earth. The graph of the $Z$ dependency on the natural coordinate radius $r$ is shown in Figures 3 and a2. 


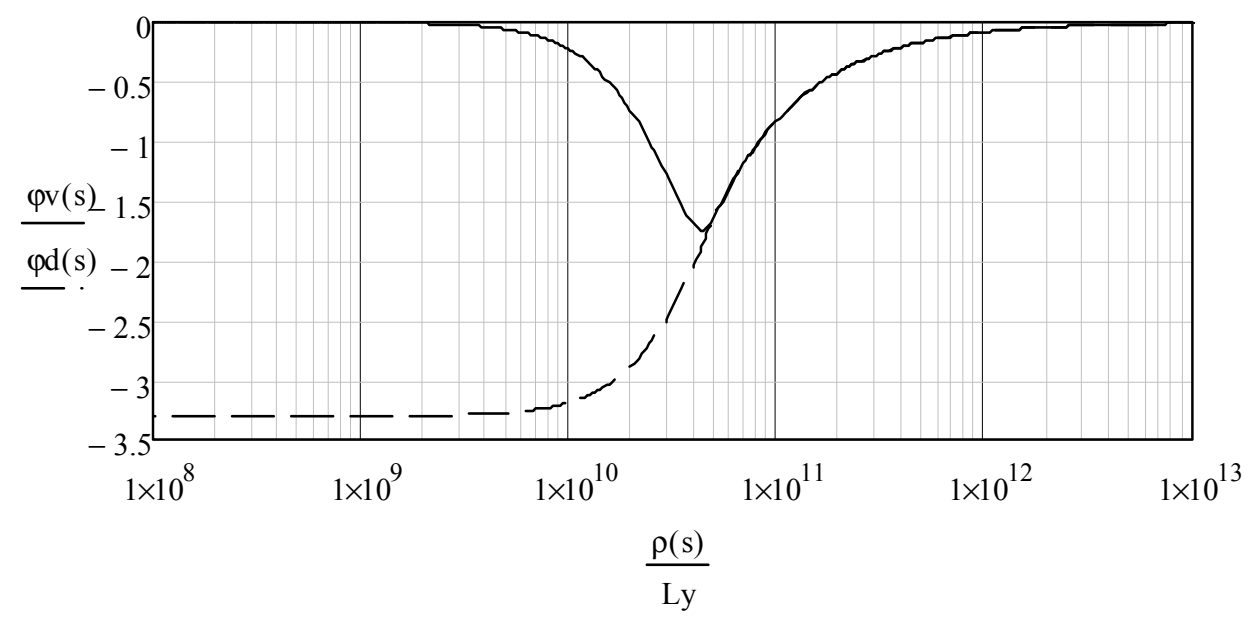

Figure a1. The graph of the dependency of normalized gravitational potential for the visible matter (solid trace) and the graph of the dependency of normalized gravitational potential for the dark matter both as functions of the physical radius. The integration constants were adjusted such that the potentials at infinity are zero

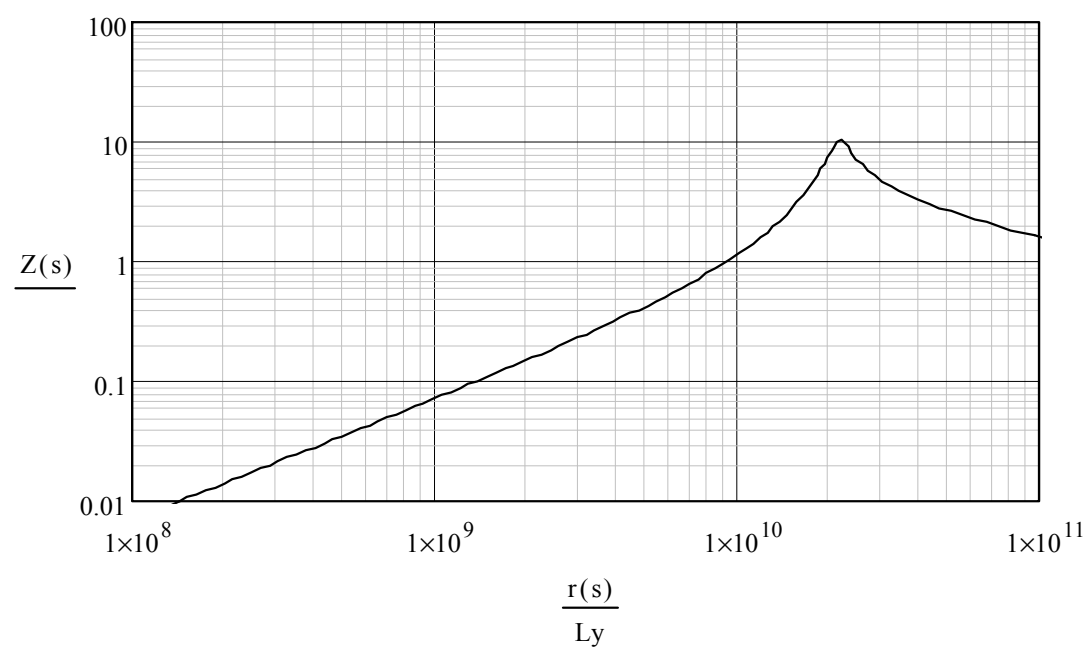

Figure a2. The graph of the dependency of redshift $Z$ on the distance $r$ measured in light years. The maximum redshift that can be observed is: $Z_{\mathrm{mx}}=10.35$. The visible matter does not exist at the distances larger than $r_{m x}=22.11 \cdot 10^{9} \mathrm{Ly}$, since it disintegrates a the Universe's edge

The radial distance $r$, used in Equation a10 and in the graph in Figure a2, also called in this paper the natural radial distance, which is the observable parameter, is calculated according to Equation a3 as follows:

$$
r(\rho)=\int_{0}^{\rho} \exp \left(\varphi_{v}\right) d \rho
$$

\section{Copyrights}

Copyright for this article is retained by the author(s), with first publication rights granted to the journal.

This is an open-access article distributed under the terms and conditions of the Creative Commons Attribution license (http://creativecommons.org/licenses/by/3.0/). 